\title{
Terahertz-to-Optical Conversion Using a Plasmonic Modulator
}

S. Ummethala ${ }^{1,2, *}$, T. Harter ${ }^{1,2}$, K. Köhnle ${ }^{1,2}$, S. Muehlbrandt ${ }^{1,2}$, Y. Kutuvantavida ${ }^{1,2}$, J. N. Kemal ${ }^{1}$, J. Schaefer ${ }^{4}$, H. Massler ${ }^{5}$, A. Tessmann ${ }^{5}$, S. K. Garlapati ${ }^{3}$, A. Bacher ${ }^{2}$, L. Hahn², M. Walther ${ }^{5}$, T. Zwick ${ }^{4}$, S. Randel ${ }^{1}$, W. Freude ${ }^{1}$, C. Koos $^{1,2, * *}$

${ }^{1}$ Institute of Photonics and Quantum Electronics (IPQ), ${ }^{2}$ Institute of Microstructure Technology (IMT), ${ }^{3}$ Institute of Nanotechonology (INT),

${ }^{4}$ Institute of Radio Frequency Engineering and Electronics (IHE), Karlsruhe Institute of Technology (KIT), 76131 Karlsruhe, Germany ${ }^{5}$ Fraunhofer Institute for Applied Solid State Physics (IAF), 79108 Freiburg, Germany sandeep.ummethala@kit.edu, ${ }^{*}$ ch hristian.koos@kit.edu

Abstract: We show a THz plasmonic modulator with flat frequency response from $40 \mathrm{MHz}$ to $0.325 \mathrm{THz}$ and employ it to demonstrate THz-to-optical conversion of a $30 \mathrm{Gbit} / \mathrm{s}$ signal on a $0.294 \mathrm{THz}$ carrier.

OCIS codes: (250.5403) Plasmonics; (060.5625) Radio frequency photonics; (130.4110) Modulators

\section{Introduction}

Global wireless network traffic increased by more than $60 \%$ in 2016 [1] and will continue to grow exponentially. Sustaining this trend will soon require wireless links that can handle data rates of up to tens of Gbit/s [2]. In this context, transmission windows of low atmospheric attenuation above $0.275 \mathrm{THz}$ are of particular interest as they offer large unallocated bandwidths for communication in pico cells (reach $<200 \mathrm{~m}$ ) and femto cells $(\mathrm{reach}<10 \mathrm{~m})$ [2]. On the technological level, integration of such wireless networks into existing fiber-optic links is a key challenge requiring novel device and signal processing concepts for seamless conversion of data streams between the $\mathrm{THz}$ and optical domains. For direct optical-to- $\mathrm{THz}(\mathrm{O} / \mathrm{T})$ conversion, optoelectronic wireless transmitters have been demonstrated, exploiting photomixing in ultra-fast uni-travelling carrier photodiodes (UTC-PD) [3] or plasmonic devices [4], thereby offering wideband tunability of the carrier frequency and leverage of advanced optical transmitter technologies. In contrast, direct THz-to-optical (T/O) conversion which offers similar advantages at the receiver is much less explored. The previous demonstrations were limited to up-conversion of unmodulated carrier tone at a frequency of $60 \mathrm{GHz}$ [5].

In this paper, we report on the first demonstration of $\mathrm{T} / \mathrm{O}$ conversion of data signals. We exploit ultra-broadband plasmonic organic hybrid $(\mathrm{POH})$ modulators with an unprecedented bandwidth of at least $0.325 \mathrm{THz}$ that can be integrated on the silicon photonic platform. These devices are combined with InP-based millimeter-wave monolithic integrated circuits (MMIC) for broadband amplification of the THz data signal. In a proof-of-concept experiment, we perform T/O conversion of a $30 \mathrm{Gbit} / \mathrm{s}$ quadrature phase shift keying (QPSK) data stream on a $0.294 \mathrm{THz}$ carrier and coherently detect the optical signal.

\section{Device Principle}

Figure 1(a) shows the top view of a plasmonic organic hybrid (POH) device in Mach-Zehnder modulator (MZM) configuration fabricated on the silicon photonic ( $\mathrm{SiP}$ ) platform. Each arm contains a phase modulator comprising a narrow metallic slot (width $75 \mathrm{~nm}$ ) between two gold electrodes (yellow). The POH MZM is configured to operate in push-pull mode. Light is coupled in and out of the SiP chip via shallow-etched grating couplers and propagates in silicon strip waveguides (green) with dimensions of $500 \mathrm{~nm} \times 220 \mathrm{~nm}$. A multimode interference (MMI) coupler splits the light and launches it into both arms of the MZM, which have a geometrical length difference of $80 \mu \mathrm{m}$ for selecting the operating point by tuning the wavelength. Tapered silicon waveguides are used for efficient conversion between silicon photonic mode and the surface plasmon polariton (SPP) that travels in each metallic slot. For EO modulation, the slots of the MZM are filled with an electro-optic (EO) polymer (SEO 100 with a bulk $r_{33}$ of 166 $\mathrm{pm} / \mathrm{V}$ ). A drive voltage applied across the ground-signal-ground (GSG) contacts of the MZM modulates the phase of the SPP wave and hence the intensity at the output of the MZM.

\section{Bandwidth Measurement}

For characterizing the frequency response of the POH MZM, we measure the EO response of the device in the frequency range $40 \mathrm{MHz} \leq f_{\bmod } \leq 0.325 \mathrm{THz}$. We investigate a modulator with a slot width of $75 \mathrm{~nm}$ and a phase shifter length of $15 \mu \mathrm{m}$. A continuous wave (CW) carrier at $1541 \mathrm{~nm}$ is launched into the POH MZM, which is driven with a small sinusoidal RF signal at the modulator's $3 \mathrm{~dB}$ point. The spectrum shows a peak at the optical carrier frequency $f_{\mathrm{c}}$ along with two first-order sidebands at $f_{\mathrm{c}} \pm f_{\text {mod. }}$. The sideband-to-carrier ratio $R_{1,0}$ allows to calculate the phase modulation index $\eta$ (assumed to be identical in both arms) according to the relation $R_{1,0} \simeq \eta^{2} / 4$ for $\eta \ll 1[6]$. The frequency response of the modulator is plotted in Figure 1(b) after normalizing $\eta$ with respect to the RF drive voltage at each frequency point. We employ three different sources for the drive signals. From $40 \mathrm{MHz}$ to $65 \mathrm{GHz}$, a vector network analyzer (VNA, Anritsu 37397C) is used. The range from $70 \mathrm{GHz}$ to $0.11 \mathrm{THz}$ and from $0.11 \mathrm{THz}$ to $0.17 \mathrm{THz}$, is covered by a Keysight VNA (PNA-X, N5247) with a suitable frequency multiplier. Frequencies from $0.22 \mathrm{THz}$ to $0.325 \mathrm{THz}$ are generated by a UTC-PD which responds to the superposition of two detuned CW lasers. We have no access to the frequencies from $0.17 \mathrm{THz}$ to $0.22 \mathrm{THz}$ in our lab. An RF probe, designated for the respective frequency bands, is used to contact the GSG pads of the MZM. The measurements from appropriate power meters and the losses from the data sheets of the RF components (cables, probes etc.) are taken into account to accurately normalize the measurements over the entire frequency range. As can be observed from the Figure 1(b), the response 
of the POH MZM is flat up to a frequency of $0.325 \mathrm{THz}$ and no indication of a roll-off can be seen. To the best of our knowledge, this is the fastest MZM demonstrated so far on the SiP platform. The measured bandwidth exceeds by far the frequency of $170 \mathrm{GHz}$, which was previously reported for POH devices [7]. The current results support the fact that the $\mathrm{POH}$ modulators have cut-off frequencies in the THz range [8].
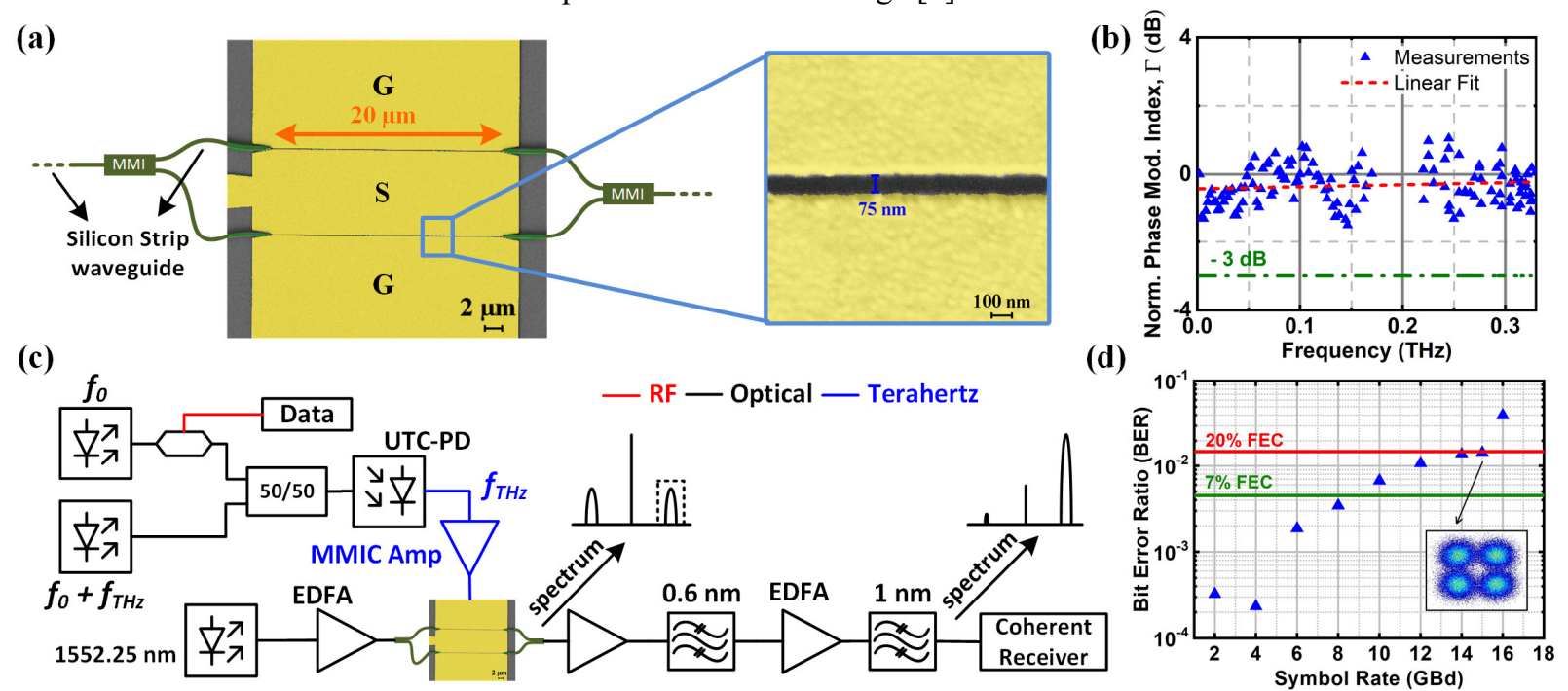

Figure 1: Ultra-broadband Plasmonic Modulator (a) False color scanning electron micrograph of plasmonic MZM with a zoom-in showing the slot width of $75 \mathrm{~nm}$. (b) Frequency response of POH MZM up to $0.325 \mathrm{THz}$. (c) Experimental setup of the terahertz-to-optical (T/O) conversion. (d) Bit error ratio (BER) of T/O conversion of QPSK signal for various symbol rates on a $0.294 \mathrm{THz}$ carrier. Inset - QPSK constellation of a $15 \mathrm{GBd} \mathrm{T} / \mathrm{O}$ converted signal.

\section{Terahertz-to-optical Conversion}

To evaluate the performance of the plasmonic modulator in THz networks, data transmission experiments are performed at a carrier frequency of $0.294 \mathrm{THz}$. A POH MZM with a phase shifter length of $20 \mu \mathrm{m}$ is used for the data experiment as shown in the Figure 1(c). An optical CW carrier a frequency $f_{0}$ is modulated with a QPSK signal and combined with a detuned $\mathrm{CW}$ tone at $f_{0}+f_{\mathrm{THz}}$ with a frequency offset of $0.294 \mathrm{THz}$. The superimposed signal is photomixed in a UTC-PD that transfers the modulation from an optical carrier to a THz carrier, $f_{\mathrm{THz}}=0.294 \mathrm{THz}$. The THz data signal is then boosted by an MMIC amplifier before being applied to the GSG pads of the POH MZM via a THz probe. The POH MZM modulates a CW laser at a wavelength of $1552.25 \mathrm{~nm}$ that is boosted to an optical power of $21 \mathrm{dBm}$ by an erbium-doped fiber amplifier (EDFA). The resulting modulated signal that is offset from the optical carrier by the $\mathrm{THz}$ carrier frequency $f_{\mathrm{THz}}$ is analyzed in a coherent receiver after a two-stage amplification and band-pass filtering to suppress the optical carrier and one of the modulation sidebands. Figure 1(d) shows the measured bit error ratio (BER) for data transmission at different symbol rates of the QPSK signal. For line rates up to $16 \mathrm{Gbit} / \mathrm{s}$, we measure a BER below the threshold of forward-error correction (FEC) with a $7 \%$ overhead. For modulation with a line rate of $30 \mathrm{Gbit} / \mathrm{s}$, we obtain a BER of $1.5 \times 10^{-2}$ which is below the threshold of FEC with a $20 \%$ overhead. The drop in the signal quality for higher line rates is due to the decrease in $\mathrm{THz}$ power and does not reflect the performance of the plasmonic modulator.

\section{Summary}

With a POH MZM having an unprecedented bandwidth of more than $0.325 \mathrm{THz}$, we demonstrate T/O conversion of a $30 \mathrm{Gbit} / \mathrm{s}$ QPSK signal on a $0.294 \mathrm{THz}$ carrier. To the best of our knowledge, this is the first demonstration of T/O data conversion.

Acknowledgment and Funding: We thank Jingdong Luo and Alex K.-Y. Jen from Soluxra for providing the organic EO material. We acknowledge support from the Alfried Krupp von Bohlen und Halbach Foundation, the European Research Council (ERC Starting Grant 'EnTeraPIC'); the Helmholtz International Research School for Teratronics (HIRST); and Karlsruhe Nano-Micro Facility (KNMF).

\section{References}

[1] Cisco, "Cisco Visual Networking Index: Forecast and Methodology, 2016-2021," White Paper, 2017.

[2] T. Kürner and S. Priebe, "Towards THz communications - Status in research, standardization and regulation," J. Infrared, Millimeter, Terahertz Waves, vol. 35, no. 1, pp. 53-62, 2014.

[3] S. Koenig et al.,"Wireless sub-THz communication system with high data rate," Nature Photon., vol. 7, no. 12, Oct. 2013.

[4] T. Harter et al., "Silicon-Plasmonic Photomixer for Generation and Homodyne Reception of Continuous-Wave THz Radiation," CLEO, SM4E.5, 2016.

[5] Y. Salamin et al., "Direct Conversion of Free Space Millimeter
Waves to Optical Domain by Plasmonic Modulator Antenna," Nano Letters, 15 (12), 8342-8346, 2015.

[6] Y. Shi, L. Yan, and a E. Willner, "High-speed electrooptic modulator characterization using optical spectrum analysis," $J$. Light. Technol., vol. 21, no. 10, 2003. Eq. (11) for $m=\eta, \eta \ll 1$

[7] C. Hoessbacher et al., "Plasmonic modulator with \&gt; $170 \mathrm{GHz}$ bandwidth demonstrated at $100 \mathrm{GBd}$ NRZ," Opt. Express, vol. 25, no. 3, p. $1762,2017$.

[8] C. Koos et al., "Silicon-Organic Hybrid ( $\mathrm{SOH}$ ) and Plasmonic Organic Hybrid (POH) Integration," J. Light. Technol., vol. 34, no. 2 , pp. 256-268, 2016. 